\title{
Simulação neutrônica do reator nuclear de pesquisa TRIGA, do CDTN, utilizando os códigos Monte Carlo Serpent e MCNPX
}

\author{
Neutronic simulation of the nuclear research reactor TRIGA of the \\ CDTN using the Monte Carlo Serpent and MCNPX Codes
}

\author{
Sincler Meireles de \\ Peixoto \\ sinclercdtn@hotmail.com \\ CDTN/CNEN
}

\section{Amir Zacarias Mesquita amir@cdtn.br CDTN/CNEN}

Maria Ângela de B. C. Menezes menezes@cdtn.br CDTN/CNEN

Maritza Rodriguez Gual maritzargual@gmail.com CDTN/CNEN

\section{Daniel Artur Pinheiro Palma dapalma@cnen.gov.br CNEN}

\begin{abstract}
Resumo
O reator nuclear de pesquisa TRIGA IPR-R1 está localizado no Centro de Desenvolvimento de Tecnologia Nuclear - CDTN, em Belo Horizonte. Ele permanece em operação desde 1960. Durante esse período, o reator tem sido utilizado na produção de radioisótopos, para estudos em radiobiologia, para produção de fontes radioativas para indústrias, na produção de traçadores utilizados em processos industriais, na irradiação de amostras para análise por ativação por nêutrons, docência e treinamento. Devido a um histórico muito longo de operações e à ausência de informações sobre a composição atual dos elementos combustíveis, torna-se muito difícil conhecer, com exatidão, seus parâmetros neutrônicos. Este trabalho apresenta a avaliação teórica do fator de multiplicação de nêutrons $(k)$ desde a primeira configuração, levando em consideração os efeitos da incerteza na composição atual do combustível do reator. Os resultados mostraram que a geometria e detalhes do reator devem ser incluídos no modelo, pois impactam significativamente no cálculo da reatividade. Comparações feitas entre os códigos Monte Carlo MCNPX e Serpent 2.1.20, mostraram diferenças consistentes dos resultados obtidos para a reatividade em todas as geometrias simuladas.
\end{abstract}

Palavras-chave: Reator. TRIGA. Nêutron. Código Serpent. MCNP.

\begin{abstract}
The IPR-R1 TRIGA nuclear research reactor is located at the Nuclear Technology Development Center - CDTN in Belo Horizonte. It has been in operation since 1960. During this period, the reactor has been used for production of radioisotopes aiming at radiobiology and environment studies, for production of radioactive sources for industries, in production of radiotracers for industrial processes, in application of neutron activation analysis and training. Due to a very long history of operations, and the absence of information about the current fuel elements composition, it becomes very difficult to know exactly its neutron parameters. This paper presents the theoretical evaluation of the neutron multiplication factor $(k)$ from the first configuration, taking into account the effects of the uncertainty in the current reactor fuel composition. The results showed that the geometry and details of the reactor must be included in the model, since it has a significant impact on the reactivity calculation. Comparisons made between the Monte Carlo codes: MCNPX and Serpent 2.1.20, showed consistent differences in the results obtained for reactivity in all simulated geometries.
\end{abstract}

Keywords: Nuclear reactor. TRIGA. Neutron. Serpent Code. MCNP.

\section{Introdução}

Os reatores de pesquisa geralmente desempenham um papel importante numa série de atividades relacionadas com a investigação e formação de pessoal, tais como a produção de radioisótopos, análise por ativação por nêutrons e experimentos em física de nêutrons e termohidráulica. Além disso, os reatores de pesquisa podem fornecer conhecimentos valiosos para testar e validar códigos computacionais (softwares) para cálculo da física de reatores nucleares.

O reator de pesquisa TRIGA IPR-R1, mostrado na Fig. 1, foi adquirido pelo governo de Minas Gerais em 1960, através do programa do governo americano "Átomos pela Paz". Localizado no Instituto de Pesquisas Radioativas (IPR), atualmente Centro de Desenvolvimento de Tecnologia Nuclear (CDTN), ele permanece em operação até os dias atuais. 
Sua primeira criticalidade ocorreu em 06 de novembro de 1960, com uma potência térmica máxima de $30 \mathrm{~kW}$. Posteriormente, acrescentaram-se mais elementos combustíveis ao núcleo, aumentando a potência para $100 \mathrm{~kW}$, sendo esta a atual potência máxima licenciada. Em 2004 foram realizadas modificações no núcleo e acrescentaram-se novos elementos combustíveis, permitindo que a potência atinja níveis de $250 \mathrm{~kW}$ (MESQUITA, 2005). Nesta época, iniciaramse estudos e experimentos, na potência de $250 \mathrm{~kW}$, para avaliar a distribuição do fluxo de nêutrons e a transferência de calor do núcleo, conduzidos por Dalle(2005) e Mesquita et al. $(2005,2007)$. Atualmente, o núcleo tem uma configuração cilíndrica com 63 elementos combustíveis cilíndricos, com $20 \%$ de enriquecimento em U-235 e 8,5\% em peso de urânio. O combustível é uma liga de hidreto de zircônio e urânio (moderador de nêutrons e combustível). Os elementos combustíveis estão fixados no núcleo e espaçados entre si por meio de duas placas de alumínio, uma superior e uma inferior. A água entra no canal pelos orifícios da placa inferior, percorre a região ativa retirando o calor do elemento combustível, sai do canal nos espaços existentes entre as cabeças dos elementos e a placa superior.

A Figura 1 mostra duas fotos do reator em operação. À esquerda tem-se uma vista do topo do poço do reator. Os cilindros, em amarelo, são os mecanismos de acionamento das barras de controle, os quais estão sobre a viga central. Esta viga suporta também o tubo central, os detectores de radiação, o tubo que leva amostras até a mesa giratória e outros equipamentos. Na foto à direita tem-se uma visão do núcleo do reator. Pode-se ver a parte superior dos elementos combustíveis (formas triangulares) afixadas na placa superior do núcleo. Veem-se também as guias das barras de controle (tubos mais finos saindo do centro do núcleo) e o tubo central (de maior espessura). A cor azul, em ambas as imagens, é característica de reatores nucleares, deve-se à radiação de Cherenkov, nome em homenagem ao cientista soviético Pavel Cherenkov que foi o primeiro pesquisador que caracterizou rigorosamente o efeito. Este efeito ocorre quando uma partícula atravessa um meio, com velocidade maior que a velocidade da luz nesse meio. Neste caso, a água.

Figura 1 - Poço e núcleo do reator nuclear de pesquisa TRIGA IPRR-1.
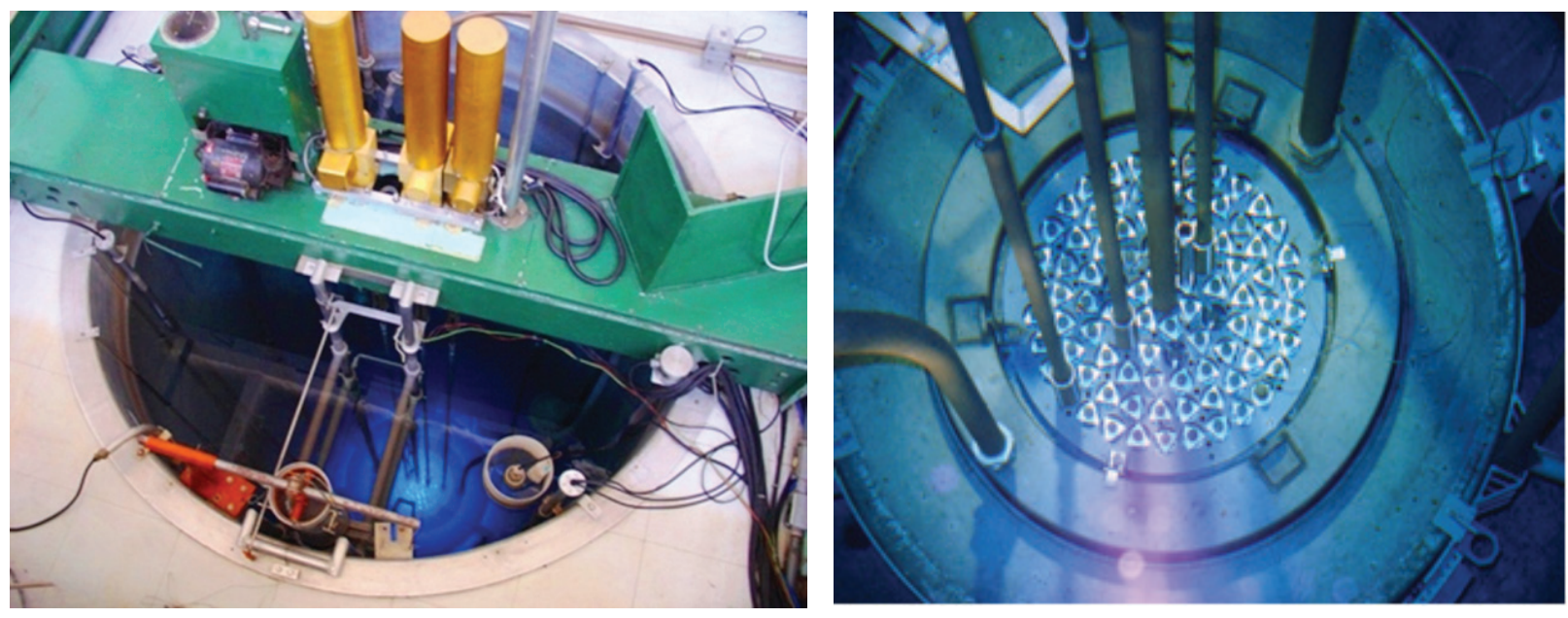

Fonte: Mesquita et al. (2016).

A Figura 2 mostra o diagrama do núcleo atual do reator IPR-R1. Ele é formado por um reticulado cilíndrico com 91 posições, onde estão instalados os 63 elementos combustíveis-moderadores (59 com revestimento de alumínio e 4 com revestimento de aço inoxidável), 23 elementos falsos de grafita, uma fonte de nêutrons, um tubo central de irradiação e três barras de controle. Esses componentes são dispostos formando anéis concêntricos e são mantidos em posição vertical, suportados por duas grades circulares. O núcleo é circundado por um refletor de grafita. Uma coluna d'água de aproximadamente $5 \mathrm{~m}$ faz a blindagem em relação ao topo do poço. A grade superior do núcleo apoia-se no topo do revestimento do refletor e possui furos circulares que permitem a passagem dos elementos combustíveis, cujas cabeças com espaçadores possibilitam a circulação da água de refrigeração através dos vazios existentes. A grade inferior apoia-se na parte inferior do revestimento do refletor. A grade inferior sustenta o peso completo do núcleo, possui orifícios para o apoio dos componentes do núcleo e furos para a entrada da água de refrigeração. 
Figura 2 - Configuração do núcleo do reator TRIGA IPR-R1.

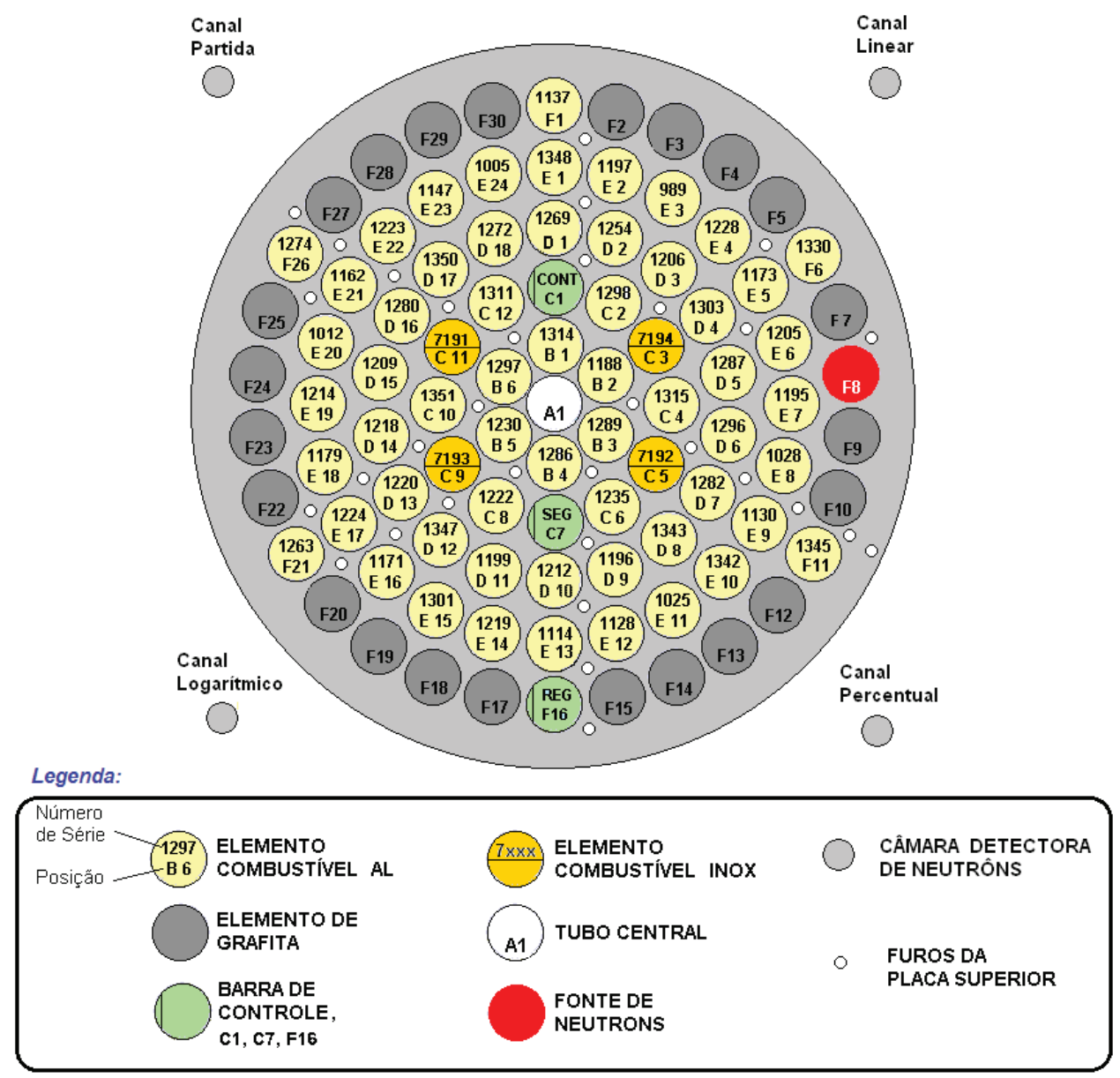

Fonte: Mesquitaet al. (2011).

Todos os trabalhos realizados com o reator dependem de dados do fluxo e da temperatura dos nêutrons, bem como de outros parâmetros. Esses dados são extremamente importantes, especialmente quando se está aplicando a análise por ativação neutrônicavia método $k_{0}$ padronizado (DE CORTE, 2006). O método é uma rotina no Laboratório de Ativação Neutrônica do CDTN (MENEZES e JAĆIMOVIĆ, 2006). Portanto, a validação dos parâmetros é de vital importância para garantir a confiabilidade das atividades desenvolvidas, sendo também uma indicação de segurança, pois aponta possíveis problemas com o reator.

Várias técnicas podem ser utilizadas para se calcular os diversos parâmetros de interesse em um reator nuclear. Os primeiros modelos para cálculo eram mais simples e consideravam o reator como um cilindro de composição uniforme. Com o passar do tempo, novos conjuntos de equações começaram a surgir para resolver a física de nêutrons. Mais recentemente esse conjunto de equações deu origem aos métodos determinísticos.

As técnicas de amostragem estatística, conhecidas hoje como método Monte Carlo, já eram conhecidas há séculos, entretanto essas técnicas haviam caído no esquecimento por causa da grande quantidade de cálculos manuais. Essas técnicas só ressurgiram durante a $2^{\mathrm{a}}$ guerra mundial, quando o matemático Stanislaw Ulam ${ }^{1}$ realizou uma visita ao Laboratório da Universidade da Pensilvânia. Nessa visita, Stanislaw ficou impressionado com a velocidade e versatilidade do ENIAC, primeiro computador eletrônico (Electronic Numerical Integrator and Computer). Assim, mediante a velocidade de cálculo do ENIAC, ocorreu a Stanislaw que estudos dessas técnicas deveriam ser reiniciados, e discutiu

\footnotetext{
${ }^{1}$ StanisławUlam matemático polonês. Participou do Projeto Manhattan, responsável pelo desenvolvimento das primeiras bombas atômicas.
} 
a ideia com o grande matemático John Von Neumann². Neumann percebeu a relevância da sugestão de Ulam e, em 1947, enviou uma carta para Robert Richtmyer, o líder da Divisão Teórica no laboratório de Los Alamos, que foi um dos laboratórios participantes da construção das primeiras bombas atômicas. A carta incluía um esboço detalhado de uma possível abordagem estatística para resolver o problema da difusão de nêutrons em material físsil (ECKHARDT e ULAM, 1987; METROPOLIS e ULAM, 1949, METROPOLIS, 1987). Foi a partir desse trabalho que essa técnica passou a ser conhecida como método Monte Carlo, nome inspirado em um tio de Ulam que jogava constantemente em um Cassino de Monte Carlo, cujo aspecto aleatório de suas roletas estava intimamente ligado ao método.

Hoje, a técnica Monte Carlo é aplicada em diversas áreas da pesquisa e tornou-se uma ferramenta matemática comumente utilizada para simular problemas que podem ser representados por processos estocásticos (YORIYAZ, 2009). O método pode então ser empregado para simular teoricamente o processo fundamentalmente estatístico da interação de partículas nucleares com a matéria, sendo particularmente usado para problemas complexos que não podem ser modelados por códigos computacionais que adotam métodos determinísticos.

Trabalhos anteriores, utilizando os códigos Monte Carlo para simulação do reator nuclear de pesquisa TRIGA IPR-R1, foram realizados por Dalle (1999, 2005), Guerra (2011), Salomé (2011) e Salomé et al.(2014). Dalle (2005) focou na determinação da queima de combustível de 1960 a 2004, considerando uma geometria simplificada para o núcleo do reator. Guerra (2011) detalhou mais o núcleo do reator, determinando os fluxos de nêutrons em posições de irradiação na mesa giratória. Salomé (2011) e Saloméet al. (2014) também estudaram os fluxos de nêutrons em posições da mesa giratória, assumindo a configuração do núcleo no modelo proposto por Guerra (2011). Entretanto, investigações adicionais se tornaram necessárias.

O foco no estudo aqui apresentado é a modelagem do reator TRIGA IPR-R1 utilizando diferentes códigos Monte Carlo de transporte de partículas: MCNPX e Serpent (HENDRICKS, 2008; BROW, 2010; LEPPÄNEN et al. 2014). A influência da geometria nos parâmetros neutrônicos dos modelos foi estudada simulando três geometrias progressivamente detalhadas. A primeira configuração do reator foi considerada para evitar incertezas quanto à composição do combustível presente em trabalhos de modelagem anteriores (DALLE, 2005).

\section{Metodologia}

Existe uma incerteza quanto à composição atual do combustível do reator IPR-R1. Assim, como uma estratégia para minimizar os fatores de incerteza, decidiu-se utilizar sua primeira configuração. Os dados para essa configuração, em 1960, BOL (Begin Of Life), as posições e composição de cada elemento combustível, são bem conhecidas. Foram avaliadas três geometrias diferentes para o reator. Foi realizada uma comparação entre os códigos Monte Carlo MCNPX e Serpent (HENDRICKS, 2008;KIEDROWSKI et al., 2010; LEPPÄNEN et al., 2014). Em todas assimulações utilizaramse as mesmas composições para os materiais que compõem o reator.

\subsection{Geometria}

Foram elaboradas neste trabalho três geometrias diferentes no intuito de melhorar os resultados das simulações e, assim, propor um modelo mais adequado para a simulação do reator TRIGA IPR-R1. As três geometrias, denominadas A, B, e C, são mostradas esquematicamente na Figura 3, em que as diversas cores representam diferentes materiais. Em todos os modelos a cor vermelha representa o alumínio, principal material de revestimento e estrutural do núcleo. Os retângulos azuis correspondem ao cerne do elemento combustível, e em amarelo tem-se o grafite do elemento combustível, que ajuda a refletir os nêutrons e evitando uma fuga longitudinal. Os elementos de grafita tem uma cor azul mais clara. O grafite do refletor que circula o núcleo aparece com a cor verde, e dentro do refletor a mesa giratória é preenchida com ar, que aparece com a cor verde água. Na cor laranja estão representadas as barras de controle do reator, e a água que circunda o núcleo aparece com a cor azul.

Na primeira geometria avaliada, denominada geometria A, o reator é constituído por elementos cilíndricos de combustível e um núcleo sem barras de controle, sem o tubo central e sem considerar a espessura de alumínio da mesa giratória localizada no refletor. Essa geometria é equivalente à utilizada em simulações anteriores (DALLE, 2005).

\footnotetext{
${ }^{2}$ John von Neumann contribuiu na teoria dos conjuntos, análise funcional, mecânica quântica, ciência da computação, economia, teoria dos jogos, análise numérica, hidrodinâmica das explosões, estatística e várias áreas da matemática. Participou do Projeto Manhattan.
} 
A segunda geometria avaliada, chamada geometria B, é uma geometria mais refinada pela introdução de novas estruturas. Inclui o tubo central, a guia das barras de controle, a espessura de alumínio na mesa giratória. As extremidades superior e inferior dos elementos de combustível do reator ganharam uma nova geometria, mais refinadas e mais próximas do elemento combustível real. Salomé e colaboradores (2014), já utilizaram esta geometria.

$\mathrm{Na}$ terceira geometria avaliada, denominada geometria $\mathrm{C}$, as barras de controle foram inseridas na posição fora do núcleo para reproduzir o mesmo comportamento observado em experimentos envolvendo as posições de barra. De acordo com relatório da General Atomics - GA (1986), fabricante do reator, as barras de controle têm uma forma cilíndrica e são revestidas em alumínio, suas extremidades inferiores apresentam uma forma cônica para reduzir a resistência imposta pela água durante um desligamento (as barras caem por gravidade). A ponta cônica não foi reproduzida neste modelo, porém o comprimento da barra foi mantido como descrito.

Figura 3 - Vista axiais das geometrias simuladas A, B e C.

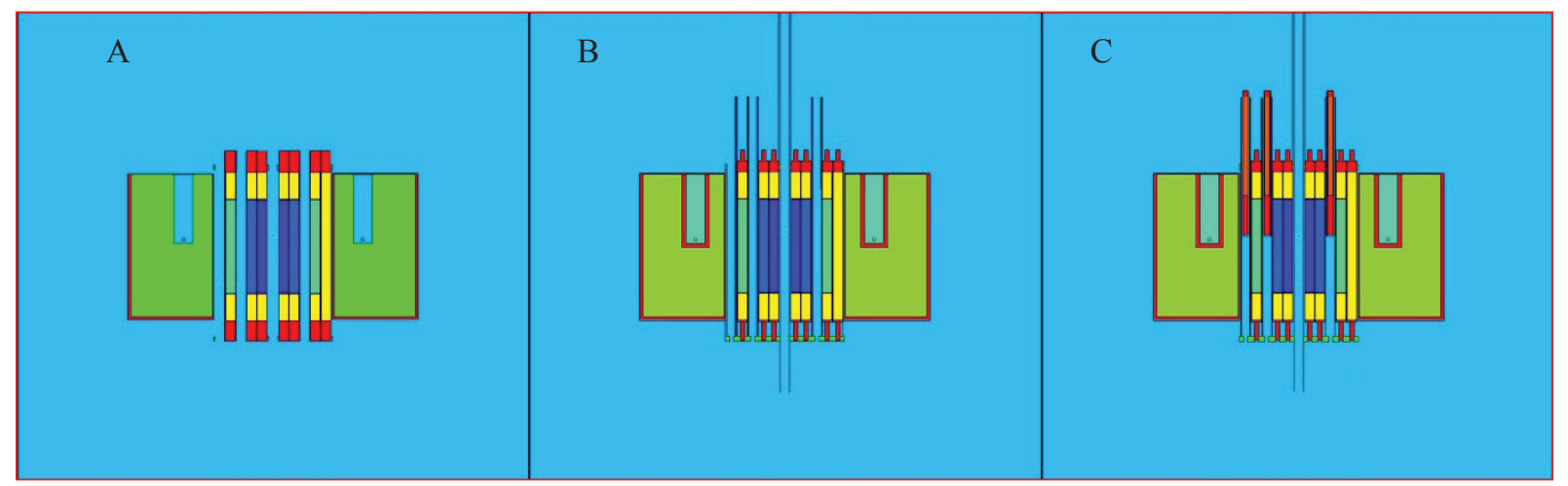

Fonte: Elaborado pelos autores.

\subsection{Os códigos Monte Carlo e as bibliotecas utilizadas}

Serpent é um código tridimensional para cálculos de criticalidade e queima de reatores utilizando a técnica Monte Carlo. Desenvolvido no Centro de Pesquisa Técnica VTT da Finlândia, em 2004, o Serpent 1 se tornou disponível ao público em 2009, sendo distribuído pelo Banco de Dados da OCDE/NEA (Organisation for Economic Co-operation and Development/Nuclear Energy Agency) e pela RSICC (Radiation Safety Information Computational Center). A nova versão do código, o Serpent 2, está atualmente em fase de testes beta. Semelhante a outros códigos baseados na técnica Monte Carlo, tais como MCNPX e Keno-VI, o Serpent usa um modelo de geometria sólida e combinatória baseado em universos, células e superfícies, que permite a descrição de praticamente qualquer configuração de combustível, ou reator bidimensional e tridimensional. A geometria consiste em células de material, definidas por superfícies elementares.

Desenvolvido pelo Laboratório Nacional de Los Alamos, o código MCNPX, que significa Monte Carlo N-Particle Extended é capaz de simular a dinâmica de diversas partículas: nêutrons, fótons, elétrons, prótons, entre outras em uma ampla faixa de energia. Podem-se simular 24 tipos de partículas. O código MCNPX não é limitado apenas a uma área específica, podendo ser utilizado para cálculos em radioproteção, dosimetria, radiografia, física médica, segurança de criticalidade nuclear, projetos e análise de detectores, design de alvos para aceleradores e projeto de reatores, descontaminação e descomissionamento (BROWet al, 2010). O código MCNPX tem sido utilizado regularmente no CDTN/CNEN, na avaliação de doses de radiação a que os operadores das instalações nucleares deste instituto de pesquisa podem receber e nos experimentos realizados (GUAL et al., 2016). Além de realizar cálculos tridimensionais, o código utiliza as bibliotecas de seção de choque mais recentes e modelos físicos para as partículas que não possuem dados tabulados disponíveis.

O sucesso da simulação com os códigos de Monte Carlo depende de escolher os melhores parâmetros para a simulação: composições atômicas dos materiais, detalhes da geometria, modelo físico, métodos de transporte, escolha correta da opção do registro (tally) e bibliotecas de seções de choque usadas pelo código que representam cada tipo de interação de radiação com a matéria. 
Bibliotecas são bancos de dados dos vários isótopos relacionando as várias seções de choque de interação com nêutrons. Expressam os vários valores da probabilidade de interação (espalhamento elástico ou inelástico, captura radiativa, emissão de partículas carregadas, reações produtoras de nêutrons e a fissão nuclear, etc.) entre um nêutron incidente e um núcleo alvo. Na física nuclear e na física das partículas, as bibliotecas de seção de choque são muito importantes quando se trata de simulações de reatores. Quando ocorre uma interação de um nêutron com um núcleo, os produtos da reação nem sempre são os mesmos e a seção de choque varia de acordo com a energia do nêutron (ou velocidade) para cada isótopo, dependendo da natureza do núcleo com o qual o nêutron interage. A maioria das interações ocorre em dois estágios. O nêutron incidente pode ser visto como absorvido pelo núcleo, formando o que se chama de núcleo composto, que pode decair de diferentes formas, podendo assim classificar as reações nêutron-núcleo segundo seus produtos da seguinte maneira: Espalhamento elástico (ou difusão), espalhamento inelástico, captura radiativa, emissão de partículas carregadas, reações produtoras de nêutrons e a fissão nuclear. A unidade padrão para medir a seção de choque é o barn, que é igual a $10^{-28} \mathrm{~m}^{2}$, ou $10^{-24} \mathrm{~cm}^{2}$.

Não existe uma expressão teórica para se calcular as secções de choque, sendo elas medidas experimentalmente. A partir de investigações experimentais é determinada a seção de choque para um determinado nuclídeo e para cada uma das diversas faixas de energia. Dessa forma, a cada novo experimento são agregados novos dados e corrigidos dados anteriores. Esse grande volume de dados sobre cada probabilidade de interação de nêutrons, para diversos isótopos e para diversas energias, forma uma biblioteca de seções de choque.

Devido à natureza experimental com que são obtidas as seções de choque, a cada nova série de experimentos tem-se uma atualização dos dados sobre as seções de choque. Tendo em vista as bibliotecas utilizadas nos trabalhos anteriores, a adoção de uma biblioteca mais recente se tornou necessária. Assim, para os testes realizados neste trabalho, a biblioteca ENDF/B-VII foi utilizada para realizar todas as simulações (CHADWICK, 2006).

Na distribuição do código Serpent são disponibilizados no pacote de utilização e instalação os dados de seções de choque baseadas na biblioteca ENDF/B-VII. Entretanto, alguns nuclídeos não têm dados definidos por essa biblioteca. Nesse caso, o programa Serpent preenche as lacunas com os dados das bibliotecas: BROND 2.2, JENDL 3.2, JEF 2.2, JEFF 3.1.1, JENDL 3.3, ou ENDF / B VI.8 (BRIESMEISTER, 2003). Para simplificação e para eliminar fatores causados pelos isótopos faltantes, foi utilizada a ENDF/B-VII, proveniente do MCNPX, em ambos os códigos.

Se os nêutrons transportados tiverem energias suficientemente baixas (tipicamente inferiores a $4 \mathrm{eV}$ ), as ligações moleculares se tornam importantes (moléculas formadas por ligações de hidrogênio, como a água e alguns sólidos cristalinos, entre outros materiais). Nesse caso, devem ser utilizados dados adicionais de seção de choque, as bibliotecas $S(\alpha, \beta)$. Essas bibliotecas são usadas para substituir as reações de dispersão elástica de gás de baixa energia para alguns núcleos de moderadores, tais como hidrogênio na água, ou carbono na grafita. Os sistemas térmicos não podem ser modelados usando seções transversais de átomo livre sem introduzir erros significativos no espectro e nos resultados.

No código Serpent 2.1.20, os dados para a liga combustível/moderador, o hidreto de zircônio, não estão disponíveis. Durante a simulação os dados foram importados do arquivo de biblioteca ENDF 70/sab, disponível para MCNPX e usado para ambos os códigos. Os materiais que utilizaram os dados de espalhamento térmico dessa biblioteca foram: água leve, hidrogênio em hidreto de zircônio, zircônio no hidreto de zircônio e grafita.

\section{Resultados e discussão}

Foram realizadas simulações da primeira configuração do reator TRIGA IPR-R1 com os códigos Serpent 2.1.20 e MCNPX com complexidades geométricas diferentes. Os primeiros testes se preocuparam em avaliar o fator de multiplicação de nêutrons $(k)$. A reação em cadeia pode ser descrita quantitativamente em termos do $k$. Ele é definido como a razão entre o número de fissões numa geração dividido pelo número de fissões na geração anterior, ou seja:

$$
K=\frac{\text { Número de fissões da geração atual }\left(N_{i}\right)}{\text { Número de fissões da geração precedente }\left(N_{i-1}\right)}
$$

$\mathrm{O}$ valor de $k$ do sistema pode ser classificado como:

$k<1$, sistema subcrítico $\Rightarrow$ não há reação em cadeia auto-sustentada.

$k=1$, sistema_crítico $\Rightarrow$ reação em cadeia no estado estacionário.

$k>1$, sistema supercrítico $\Rightarrow$ reação em cadeia crescente. 
Se o número de nêutrons de uma geração é igual ao da geração seguinte, então $k=1$ e o reator está crítico, de forma que a reação em cadeia se mantém em um nível constante. Um reator pode ficar crítico em qualquer nível de energia. Se sucessivas gerações de nêutrons provocam uma diminuição do número de fissões entre gerações, então $\mathrm{k}<1$ e o reator está subcrítico, ou seja, o nível de potência do reator será decrescente. Se sucessivas gerações de nêutrons resultam num aumento do número de fissões entre gerações, então $k>1$, o reator está super crítico e a potência do reator será crescente. O nível de potência de um reator pode alterar apenas quando o reator está subcrítico (a potência diminuirá), ou se o reator está super crítico (a potência aumentará). A manutenção da potência do reator a um valor prescrito, ou a mudança do nível de potência para um novo valor, a uma velocidade prescrita, são as funções que devem ser executadas pelo sistema de controle do reator. O sistema de controle do reator muda o valor de $k$, alterando o que acontece com os nêutrons durante o seu tempo de vida, isto é, a partir do momento em que nascem até que escapam do reator, ou são absorvidos (MESQUITA, 2017).

Para um reator crítico, a cada nêutron que nasce, exatamente um nêutron deve causar fissão na próxima geração. Há seis fatores que governam a produção, a fuga, e absorção dos nêutrons, e que permitem fazer uma descrição quantitativa dos componentes que regulam o fator de multiplicação no ciclo de nêutrons. O equilíbrio entre a produção de nêutrons e a sua absorção no núcleo, e sua fuga para fora do núcleo, determina o valor do fator de multiplicação. Se as perdas são pequenas o suficiente para serem desprezadas, o fator de multiplicação dependerá apenas do equilíbrio entre a produção e a absorção. Neste caso, ele é chamado de fator de multiplicação infinita, $k_{\infty}$ (em um núcleo infinitamente grande não haverá fugas). Quando as fugas estão incluídas, o fator é chamado de fator de multiplicação efetivo ( $k_{\text {effl). }}$ Por definição, as constantes de multiplicação $k_{e f} \mathrm{e} \mathrm{k}_{\infty}$ são números adimensionais.

O fator de multiplicação efetivo $k$ é um parâmetro que, apesar de muito simples, é de extrema importância para avaliar o comportamento de um reator nuclear. Sob condições normais de funcionamento, um reator opera na criticalidade, ou muito perto dela, isto é, $k$ é aproximadamente igual a 1,0. Pequenos desvios de $k$, acima ou abaixo de 1, irão resultar em mudanças significativas na potência do reator. Sob essas circunstâncias, é mais prático para trabalhar em termos da quantidade pelo qual $k$ se desvia da unidade, em vez de citar vários zeros antes de chegar a qualquer dígitos significativos depois de 1 . O termo que se adotou como bastante prático na análise do reator para descrever seu comportamento quando $k$ desvia de 1 é chamado de reatividade, e é o parâmetro mais importante na operação do reator nuclear. Reatividade é a medida do afastamento da posição de criticalidade em função do fator de multiplicação. Note-se que os níveis de potência, a densidade dos nêutrons, etc., estão constantemente mudando quando $k$ não é igual a 1,0. A diferença entre um dado valor de $k$ e 1,0 é denominado como o "excesso" do fator de multiplicação $(\delta k$ ), e tanto pode ser positivo como negativo, dependendo se $k$ for maior ou menor do que 1,0. Quando é positivo, o reator é supercrítico; quando é zero, ele está critico, e, quando negativo, o reator está subcrítico.

A reatividade pode ser controlada de várias formas: por adição, ou remoção, de combustível; alterando a fração de nêutrons que fogem do sistema, e ainda por alteração da quantidade do material absorvedor, que compete com o combustível na captura dos nêutrons. A reatividade é dada pelo símbolo $\rho$ e está relacionada com o acréscimo $\delta k$ e com fator de multiplicação $k$, conforme abaixo:

$$
\rho=\frac{\delta k}{1+\delta k} \rho=\frac{k-1}{k}
$$

As alterações de reatividade envolvidas nas operações normais do reator são pequenas e envolvem valores de $k$ muito próximos de 1 , por exemplo $k=1,003$ dá um $\delta k=0,003$. A reatividade é uma quantidade definida matematicamente que não pode ser medida diretamente na prática. Uma vez que $k$ é um número adimensional, a quantidade $\rho$ é um número puro. No entanto, várias unidades são utilizadas para expressar a reatividade: número puro, ou por cento (x100 número puro), ou ainda o "mk" $(1 \mathrm{mk}=0,001)$. A unidade mais comum utilizada nos reatores de pesquisa é o dólar, que é dividido em centavos. A unidade para a reatividade de uso comum nos reatores de potência é o pcm ("por cem mil"), que é igual a um valor de $\rho$ de $10^{-5}(=1 \mathrm{pcm})$. Entretanto, em reatores de pesquisa, essa unidade passou a ser utilizada atualmente pela facilidade de leitura e devido a outras dificuldades relacionadas ao uso de dólares e centavos.A unidade em pcm é usada na maioria dos reatores, porque os valores de inserção de reatividade geralmente são bastante pequenos e as unidades de pcm permitem que a reatividade seja escrita em números inteiros. As mudanças operacionais, como o 
movimento das barras de controle, causam geralmente a inserção de reatividade da ordem das unidades de pcm por um passo (MESQUITA, 2017).

Os cálculos de criticalidade foram efetivados através do cartão KCODE, disponível no código MCNPX. As simulações foram realizadas com uma população inicial de nêutrons de 10.000 nêutrons e foi amostrada para 5.000 ciclos de tempo após 1.000 passos iniciais. Os testes foram executados em sistema operacional Linux, dividido em quatro processadores. Como parâmetro de avaliação foi calculada a constante de multiplicação efetiva de nêutrons $\left(k_{e f f}\right)$, a qual pode ser calculada por métodos diferentes, sendo os valores apresentados o valor final estimado pelos métodos de colisão e absorção. Os resultados do cálculo do $k_{\text {eff }}$ e da incerteza dada pelo desvio padrão da média (DP) para a geometria A são apresentados na Tabela 1 .

Tabela 1: Comparação entre os códigos MCNPX e Serpent - Geometria A.

\begin{tabular}{c|c|c|c|c|c|c}
\hline \multirow{2}{*}{ Método } & \multicolumn{2}{|c|}{ MCNPX } & \multicolumn{2}{c|}{ Serpent $\mathbf{2 . 1 . 2 0}$} & \multicolumn{2}{c}{$\Delta \boldsymbol{k}_{\text {eff }}$} \\
\cline { 2 - 6 } & $\boldsymbol{k}_{\text {eff }}$ & $\boldsymbol{D P}$ & $\boldsymbol{k}_{\text {eff }}$ & $\boldsymbol{D P}$ & $\Delta \boldsymbol{k}_{\text {eff }}$ (pcm) & $\boldsymbol{D P}$ \\
\hline Colisão & 1,01375 & 0,00017 & 1,01215 & 0,00021 & 160 & 38 \\
Absorção & 1,01377 & 0,00012 & 1,01222 & 0,00014 & 155 & 26 \\
\hline
\end{tabular}

Fonte: Elaborada pelos autores.

Na geometria B introduziram-se as barras de controle, os tubos guias, a espessura de alumínio na mesa de amostras localizada no refletor e a nova geometria do elemento combustível. Foi inserida uma reatividade negativa em relação ao modelo inicial. $\mathrm{O}$ valor da constante de multiplicação efetiva apresentou um resultado inferior, em cerca de $470 \mathrm{pcm}$ para cada simulação. Os dados podem ser vistos na Tabela 2.

Tabela 2: Comparação entre os códigos MCNPX e Serpent - Geometria B.

\begin{tabular}{c|c|c|c|c|c|c}
\hline \multirow{2}{*}{ Método } & \multicolumn{2}{|c|}{ MCNPX } & \multicolumn{2}{c|}{ Serpent 2.1.20 } & \multicolumn{2}{c}{$\Delta \boldsymbol{k}_{\text {eff }}$} \\
\cline { 2 - 6 } & $\boldsymbol{k}_{\text {eff }}$ & $\boldsymbol{D P}$ & $\boldsymbol{k}_{\text {eff }}$ & $\boldsymbol{D P}$ & $\Delta \boldsymbol{k}_{\text {eff }}$ (pcm) & $\boldsymbol{D P}$ \\
\hline Colisão & 1,00904 & 0,00020 & 1,00728 & 0,00024 & 176 & 44 \\
Absorção & 1,00907 & 0,00014 & 1,00733 & 0,00017 & 174 & 31 \\
\hline
\end{tabular}

Fonte: Elaborada pelos autores.

A inserção de barras de controle na geometria $C$ introduziu na simulação cerca de 150 pcm de reatividade negativa quando comparada com a geometria B, o que mostra que mesmo com as barras de controle extraídas, ainda interferem na reatividade. Quando comparado com a geometria A, as alterações somam-se a uma inserção de reatividade negativa em torno de $660 \mathrm{pcm}$. Ver dados na Tabela 3.

Tabela 3: Comparação entre os códigos MCNPX e Serpent - Geometria C.

\begin{tabular}{c|c|c|c|c|c|c}
\hline \multirow{2}{*}{ Método } & \multicolumn{2}{|c|}{ MCNPX } & \multicolumn{2}{c|}{ Serpent $\mathbf{2 . 1 . 2 0}$} & \multicolumn{2}{c}{$\Delta \boldsymbol{k}_{\text {eff }}$} \\
\cline { 2 - 6 } & $\boldsymbol{k}_{\text {eff }}$ & $\boldsymbol{D P}$ & $\boldsymbol{k}_{\text {eff }}$ & $\boldsymbol{D P}$ & $\Delta \boldsymbol{k}_{\text {eff }}$ (pcm) & $\boldsymbol{D P}$ \\
\hline Colisão & 1,00755 & 0,00014 & 1,00568 & 0,00017 & 187 & 31 \\
Absorção & 1,00726 & 0,00010 & 1,00538 & 0,00012 & 188 & 22 \\
\hline
\end{tabular}

Fonte: Elaborada pelos autores.

\section{Conclusão}

Foram realizadas simulações baseadas na primeira configuração do núcleo do reator TRIGA IPR R1 utilizando os códigos MCNPX 2.0 e Serpent 2.1.20. Foram avaliados os efeitos sobre a reatividade devido ao aumento da complexidade geométrica no modelo. Os resultados mostraram que a geometria e detalhes do reator devem ser incluídos no modelo, pois tem um impacto significativo no cálculo da reatividade. 
Comparações feitas entre os códigos MCNPX 2.0 e Serpent 2.1.20 mostraram diferenças nos resultados obtidos para a reatividade em todas as geometrias simuladas. À medida que a complexidade geométrica aumentou, observou-se uma pequena redução na reatividade calculada, devido à inclusão dos materiais absorvedores presentes no tubo central, na guia das barras de controle, na mesa giratória e nas extremidades superior e inferior dos elementos de combustível do reator. Em todos os cálculos com o código Serpent foi estimado um valor menor para $k_{\text {eff }}$ comparado com o valor experimental.

Em trabalhos futuros, os efeitos de temperatura serão levados em consideração e um cálculo de queima de combustível será realizado até a data atual. Os resultados experimentais disponíveis atualmente serão utilizados para validar as simulações.

\section{Agradecimentos}

Esta pesquisa é apoiada pelas seguintes instituições: Centro de Desenvolvimento de Tecnologia Nuclear (CDTN), Comissão Nacional de Energia Nuclear (CNEN), Fundação de Apoio à Pesquisa do Estado de Minas Gerais (Fapemig), Fundação de Amparo à Pesquisa do Estado do Rio de Janeiro (Faperj), Conselho Nacional de Desenvolvimento Científico e Tecnológico (CNPq), Coordenação de Aperfeiçoamento de Pessoal de Nível Superior (Capes), Instituto Nacional de Reatores Nucleares Inovadores (INCTRNI) e Eletrobras/Eletronuclear.

\section{Referências}

BROW, F.; KIEDROWSKI, B.; BULL, J. MCNP-1.60 Release Notes. Report No. LA-UR-10e06235. Los Alamos: Los Alamos National Laboratory, 2010.

CHADWICK et. al., ENDF/B-VII.0: next generation evaluated nuclear data library for nuclear science and technology, Nuclear Data Sheets, v. 107, n. 12, p. 2931-3060, Dec. 2006.

CHARACTERIZATION of TRIGA fuel: GA Project 3442. San Diego: General Atomics Technologies, 1986.

DALLE, H. M. Avaliação do reator TRIGA IPR-R1 em operações em 250 kW. Belo Horizonte: CDTN. 1999.

DALLE, H. M. Simulação do reator TRIGA IPR-R1 utilizando o método de transporte por Monte Carlo. 2005. 189f Tese. (Doutorado em Engenharia Química) - Universidade Estadual de Campinas, Campinas. 2005.

DE CORTE, F. The k0 standardization method: a move to the optimization of reactor neutron activation analysis. Agrégé Thesis, Rijksuniversitiet Gent, 1987.

ECKHARDT, R. Stan Ulam John Von Neumann, and the Monte Carlo Method. Los Alamos Science, p 131-141, 1987. Special Issue.

GUAL, M. R.; et al. Dosimetry assessment during the sipping test in the IPR-R1 TRIGA reactor using MCNPX. Progress in Nuclear Energy, v. 93, p. 238-245, nov. 2016.

GUERRA, B. T. Obtenção do fluxo de nêutrons nos terminais de irradiação do reator TRIGA IPR-R1 usando o método de transporte Monte Carlo. 2011. 47f. Dissertação (Mestrado) - Escola de Engenharia da UFMG, Belo Horizonte, 2011.

HENDRICKS, J. S.; et. al. MCNPX 2.6.0 extensions. Technical Report LA-UR-08-2216. Los Alamos: Los Alamos National Laboratory, 2008.

KIEDROWSKI, B.; et al. 2010. MCNP-1.60 feature enhancements and manual clarifications: LA-UR-10- 06217. Los Alamos: Los Alamos National Laboratory, 2010.

LEPPÄNEN, J.; et al. Calculation of effective point kinetics parameters in the Serpent 2 Monte Carlo code. Annals of Nuclear Energy, 65, p. 272-279, 2014.

MENEZES, M. A. B. C.; JAĆIMOVIĆ R. Optimized k0-instrumental neutron activation method using the TRIGA MARK I IPR-R1 Reactor at CDTN/CNEN. Belo Horizonte: Nuclear Instruments \& Methods in Physics Research A, v. 564, 2006.

MESQUITA, A. Z.; et. al. A human-machine interface for a TRIGA research reactor of Brazil. International Journal of Nuclear Energy, Science and Technology v. 10, p. 369-384, 2016. 
MESQUITA, A. Z. Investigação experimental da distribuição de temperaturas no reator nuclear de pesquisa TRIGA IPR-R1. 2005. 168f. Tese. (Doutorado em Engenharia Química) - Universidade Estadual de Campinas, São Paulo, 2005.

MESQUITA, A. Z.; REZENDE, H. C.; FERREIRA, A. V. Monitoramento de parâmetros termohidráulicos do núcleo do reator de pesquisa TRIGA IPR-R1. Revista Tecnologia, Fortaleza, v. 32, p. 145-153, 2011.

MESQUITA, A. Z.; REZENDE, H. C.; TAMBOURGI, E. B. Calibração da potência do reator nuclear de pesquisa TRIGA IPR-R. Marzo 2005. Revista Iberoamericana de Ingeniería Mecánica, Madrid, v. 9, n.1, p. 37-45. 2005.

MESQUITA, A. Z.; REZENDE, H. C.; TAMBOURGI, E. B. Power calibration of the TRIGA mark I nuclear research reactor. Journal of the Brazilian Society of Mechanical Sciences and Engineering, Rio de Janeiro, v. 29, p. 240-245. 2007.

METROPOLIS, N. The Beginning of the Monte Carlo method. Los Alamos Science, 1987. Special Issue.

METROPOLIS, N.; ULAM, S. The Monte Carlo Method. Journal of the American Statistical Association, v. 44, p. 335-341. 1949.

SALOMÉ, J. A. D. Avaliação do fluxo em ligas Al-Au de diferentes dimensões do reator TRIGA IPR-1 usando o método Monte Carlo. 2011. 96 f. Dissertação (Mestrado em Ciências e Técnicas Nucleares) - Escola de Engenharia da UFMG, Belo Horizonte. 2011.

SALOMÉ, J. A. D.; et al. Evaluation of the thermal neutron flux in samples of Al-Au alloy irradiated in the carrousel channels of the TRIGA Mark I IPR-R1 reactor using MCNP code. Nuclear Engineering and Design. v. 273, p. 576$583,2014$.

YORIYAZ, H. Método Monte Carlo: princípios e aplicações na área de física médica. Revista Brasileira de Física Médica, p. 141-149, 2009.

Sobre os autores

\section{Sincler Peixoto de Meireles}

Graduado em Física. Pontifícia Universidade Católica de Minas Gerais - PUC MG. Mestrado e Doutorado em andamento, Programa de Ciência e Tecnologia das Radiações, Minerais e Materiais pelo Centro de Desenvolvimento da Tecnologia Nuclear - CDTN.

\section{Amir Zacarias Mesquita}

Engenheiro Eletricista, Universidade Federal de Minas Gerais - UFMG. Mestre em Ciências e Tecnologia Nuclear, Universidade Federal de Minas Gerais - UFMG. Doutor em Engenharia Química, Universidade Estadual de Campinas - UNICAMP. Pesquisador Titular III do Centro de Desenvolvimento da Tecnologia Nuclear da Comissão Nacional de Energia Nuclear - CDTN/CNEN.

\section{Maria Ângela de Barros Correia Menezes}

Engenheira Química, Universidade Federal de Minas Gerais. Mestre em Ciências e Técnicas Nucleares, Universidade Federal de Minas Gerais - UFMG. Doutora em Química (Inorgânica) pela Universidade Federal de Minas Gerais - UFMG, Pesquisadora Titular III do Centro de Desenvolvimento da Tecnologia Nuclear da Comissão Nacional de Energia Nuclear - CDTN/CNEN.

\section{Maritza Rodriguez Gual}

Graduação em Engenharia Energética Nuclear, "Faculdade de Ciências y Tecnologia Nuclear" da Universidade Havana, Cuba. Mestre em Técnicas de Reatores Nucleares, Universidade Técnica de Budapeste - Hungria. Doutor em Ciências Técnicas Nucleares, "Instituto Superior de Tecnologias y Ciências Aplicadas" - InSTEC, Cuba. Pós-Doutorado em andamento, Programa em Ciência e Tecnologia das Radiações, Minerais e Materiais pelo Centro de Desenvolvimento da Tecnologia Nuclear - CDTN.

\section{Daniel Artur Pinheiro Palma}

Graduação em Física, Universidade Federal do Rio de Janeiro. Mestre em Física pela Universidade Federal do Rio de Janeiro. Doutor em Engenharia Nuclear pela Coordenação dos Programas de Pós-graduação de Engenharia (COPPE/ UFRJ). Tecnologista Pleno III/III da Comissão Nacional de Energia Nuclear. 